\title{
New host records for Zeuxia cinerea Meigen, 1826 (Diptera: Tachinidae) from Turkey
}

$$
\text { Neslihan GÜLTEKİN }{ }^{1} \text {, Celalettin GÖZÜAÇIK }{ }^{1 *} \text {, Kenan KARA², Turgut ATAY }
$$

ABSTRACT: The paper presents new host records for Zeuxia cinerea Meigen, 1826 (Diptera: Tachinidae) from Turkey. New parasitoid host couples are Temnorhinus hololeucus (Pallas, 1781) and Maximus strabus (Gyllenhal, 1834) (Coleoptera: Curculionidae) for Z. cinerea. Some additional information about the reared species and hosts are also presented.

Key Words: Zeuxia cinerea, new host records, Turkey.

\footnotetext{
${ }^{1}$ Neslihan GÜLTEKİN (Orcid ID: 0000-0002-0139-739, Celalettin GÖZÜAÇIK (Orcid ID: 0000-0002-6543-7663), Iğdır University, Faculty of Agriculture, Department of Plant Protection, Iğdır, Turkey

2 Kenan KARA (Orcid ID: 0000-0003-0439-5639), Turgut ATAY (Orcid ID: 0000-0002-9074-0816), Gaziosmanpaşa University, Faculty of Agriculture, Department of Plant Protection, Tokat, Turkey

*Sorumlu Yazar/Corresponding Author: Celalettin GÖZÜAÇIK, e-mail: cgozuacik46@gmail.com
} 


\section{INTRODUCTION}

All of the known species of Tachinidae (Diptera) are parasitoids of insects or other arthropods (Stireman et al., 2006). Knowledge on their biology, including host insect species is not revealed yet, so every host-couple record is crucial.

The host-parasitoid couples of Turkish tachinids is not well known. New host-parasitoid couples are done every year. Both faunistic and host parasitoid studies related to tachinids need to be done in Turkey. Host catalogue of Turkish Tachinidae by Kara and Tschorsnig (2003) and Checklist of Turkish Tachinidae is published by (Kara et al., 2020).

The species of the Zeuxia only were reared from mostly Curculionidae and Cerambycidae and most rarely Cleridae (Coleoptera). Zeuxia cinerea has a narrow host range. Curculionidae (Coleoptera) is the usual host family of this tachinid (Tschorsnig, 2017). Previously, Zeuxia cinerea were reared from Larinus sibiricus Gyllenhal, 1835 (=Larinus aeruginosus Hochhuth, 1851), Larinus iaceae (Fabricius, 1775), Larinus sp., Rhinocyllus conicus (Frölich, 1792) (Col.: Curculionidae) in Turkey (Richter et al., 2002).

\section{MATERIAL AND METHODS}

Larvae of Temnorhinus hololeucus (Pallas, 1781) (six individuals) and Maximus strabus (Gyllenhal, 1834) (four individuals) were collected in Iğdır province in 2019. They were brought to the laboratory with their food-plants for rearing and transferred to separate containers (Figs 1A-B) and checked daily. Zeuxia cinerea was reared from these hosts. The key of Tschorsnig \& Herting (1994) was used for tachinid identification. Nomenclature and arrangement of tachinids follows Herting \& DelyDraskovits (1993). The specimens were deposited at the Plant Protection Museum in Gaziosmanpaşa University, Agricultural Faculty, Plant Protection Department, Tokat, Turkey.

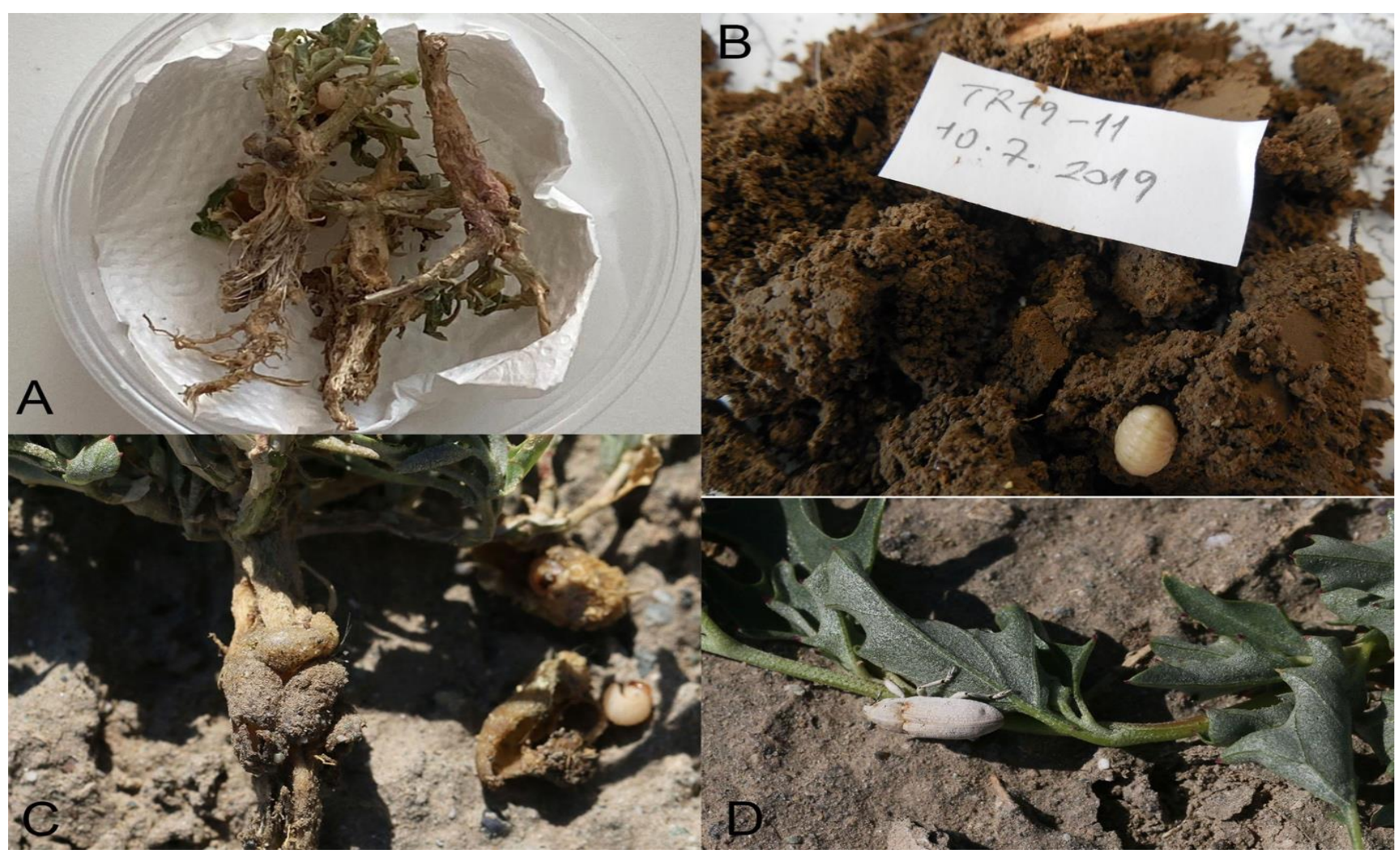

Figure 1. Rearing of parasitoids from Temnorhinus hololeucus (Pallas) and Maximus strabus Gyllenhal larvae. A-B, culture containers (A- T. hololeucus; B- M. strabus); C, larva capsule of T. hololeucus on root of Atriplex; D, adult of T. hololeucus 


\section{RESULTS AND DISCUSSION}

The distribution, hosts and some additional information related to tachinid and coleopteran hosts are as follows.

Temnorhinus hololeucus (Pallas, 1781) (Col.: Curculionidae) (Figs 1A-C-D)

Distribution: Lithuania, Russia, Azerbaijan, Armenia, China (Gansu, Xinjiang), Iran, Israel, Jordan, Kazakhistan, Mongolia, Pakistan, Turkmenistan, Turkey, Uzbekistan (Alonso-Zarazaga et al., 2017; Meregalli, 2017).

Host plants: Atriplex sp. Atriplex tatarica L., Salsola sclerantha C. A. Meyer, S. carinata C. A. Meyer, Salsola leptoclada (Ter-Minassian 1988; Fremuth 1987; Gültekin 2012). This species larvae make trehala-like capsules on root of host plant Atriplex to complete life cycle (Gültekin, 2012).

Material collected: Turkey: Iğdır Prov., 4-5 km E of Ramazankent, 3957'5.85" N, 44²7'24.80" E, 821 m, 02.07.2019, N. Gültekin and C. Gözüaçık leg., 6 larvae in capsules with root crown of Atriplex sp. (Fig 1C)

Maximus strabus (Gyllenhal, 1834) (Col.: Curculionidae) (Fig 1B)

Distribution: Russia, Moldavia, Romania, Ukraine, Azerbaijan, Afghanistan, Armenia, Iran, Kazakhstan, Mongolia, Syria, Tajikistan, Turkmenistan, Turkey, Uzbekistan, China (Xinjiang) (AlonsoZarazaga et al. 2017), Australia, introduced (Meregalli, 2017).

Host plants: A Chenopodiaceae species.

Material collected: Turkey: Iğdır Prov., $6 \mathrm{~km}$ E of Tuzluca along Turabi road, 4003'24.39" N, 4344'34.82" E, 1017 m, 10.07.2019, N. Gültekin and C. Gözüaçık leg., 4 larvae in soil.

\section{Zeuxia cinerea Meigen, 1826 (Diptera: Tachinidae)}

Distribution: Palaearctic: Europe (E. Europe (Czech Republic, Hungary, Moldova, Poland, Romania, Slovakia, Ukraine), S. Europe (Albania, Bosnia \& Herzegovina, Bulgaria, Croatia, Greece, Italy, Macedonia, Portugal, Serbia, Spain), W. Europe (Austria, France, Germany, Switzerland)), Kazakhstan, Middle East (Iran, Israel, "Palestine"), North Africa (Algeria), Russia (Western Russia), Transcaucasia (Armenia) (O’Hara et al., 2019). Turkey: Tokat (Kara, 1999, Lekin, Kara, \& Atay, 2016), Erzurum (Richter et al., 2002), Karabük, Kastamonu, Zonguldak (Korkmaz, 2007), Eskişehir (Kara \& Aksu, 2007), Muğla (Lutovinovas et al, 2018).

Hosts: Conorhynchus mendicus (Gyllenhal, 1834); Larinus obtusus Gyllenhal, 1835, L. planus (Fabricius, 1792), Lixus juncii Boheman, 1835 (Coleoptera: Curculionidae) (Tschorsnig, 2017), In Turkey: Larinus sibiricus Gyllenhal, 1835 (=Larinus aeruginosus Hochhuth, 1851), Larinus iaceae (Fabricius, 1775), Larinus sp., Rhinocyllus conicus (Frölich, 1792) (Coleoptera: Curculionidae) (Richter et al., 2002).

Temnorhinus hololeucus and Maximus strabus are new host species for this tachinid.

Biology: Z. cinerea is seen from the end of May to mid-September on flowering plants and probably has 2 generations per year (Tschorsnig and Herting, 1994).

Reared specimens (date of adult emergence): 10.07.2019, $1 \lesssim$, reared from larva of Temnorhinus hololeucus, 19.07.2019, 19, reared from larva of Maximus strabus.

\section{CONCLUSION}

As a result, new host records for Zeuxia cinerea (Tachinidae: Diptera) were obtained from Turkey. These were Temnorhinus hololeucus and Maximus strabus (Coleoptera: Curculionidae). 


\section{ACKNOWLEDGEMENTS}

We are grateful to Dr. H.-P. Tschorsnig (Staatliches Museum fur Naturkunde, Stuttgart, Germany) for information on host-parasitoid couple. This study was supported by Iğdır University Scientific Research Project Council (BAP) 2019- FBE-A14 project.

\section{REFERENCES}

Alonso-Zarazaga MA, Barrios H, Borovec R, Bouchard P, Caldara R, Colonnelli E, Gültekin L, Hlaváč P, Korotyaev B, Lyal CHC., Machado A, Meregalli M, Pierotti H, Ren L, Sánchez-Ruiz M, Sforzi A, Silfverberg H, Skuhrovec J, Trýzna M., Velázquez de Castro AJ, Yunakov NN, 2017. Cooperative Catalogue of Palaearctic Coleoptera Curculionoidea. Monografías electrónicas S.E.A., vol. 8, Sociedad Entomológica Aragonesa S.E.A., Zaragoza, Spain, 729 pp.

Fremuth J, 1987. Ergebnisse der Tschechoslowakisch-Iranischen Entomologischen Expeditionen Nach Dem Iran 1970, 1973 und 1977 Coleoptera, Curculionidae; 1. Teil: Cleoninae. Acta Entomologica Musei Nationalis Pragae, 42, 321-348.

Gültekin L 2012. New Data on Conorhynchus hololeucus (Pallas, 1781) (Coleoptera: Curculionidae). Florida Entomologist 95(2): 500-502.

Herting B, Dely-Draskovits Á, 1993. Family Tachinidae. In: A., Soós, L. Papp, (Eds.). Catalogue of Palaearctic Diptera. Anthomyiidae-Tachinidae (pp.118-458), Budapest.

Kara K, 1999. Dexiinae (Diptera: Tachinidae) species of the Tokat province. Türkiye Entomoloji Dergisi, 23 (3), 203-210.

Kara K, Tschorsnig HP, 2003. Host Catalogue for the Turkish Tachinidae (Diptera). Journal of Applied Entomology, 127, 465-476.

Kara K, Aksu S, 2007. Eskişehir ve Çevresinde Belirlenen Bazı Tachinidae (Insecta: Diptera) Türleri. Türkiye II. Bitki Koruma Kongresi, 27-29 Ağustos 2007, Isparta, Turkey, 166.

Kara K, Tschorsnig HP, Atay T, 2020. Checklist of Turkish Tachinidae (Insecta, Diptera) with Journal of the Entomological Research Society, 22(2): 57-84.

Korkmaz Y, 2007. Batı Karadeniz Bölgesi Tachinidae (Hexapoda: Diptera) Türleri Üzerinde Faunistik Çalışmalar. Yüksek Lisans Tezi. Tokat Gaziosmanpaşa Üniversitesi, Fen Bilimleri Enstitüsü, Tokat, 53 (Basılmış).

Lekin N, Kara K, Atay T, 2016. Tachinidae (Diptera) Species From Some Uplands in Tokat Province (Turkey). Journal of Agricultural Faculty of Gaziosmanpaşa University, 33 (1), 56-63.

Lutovinovas E, Tschorsnig HP, Barták M, Kubík Š, Dursun O, Civelek HS, Kara K, 2018. Contribution to the Tachinid Fauna of Southwestern Turkey (Diptera: Tachinidae). Annales de la Société entomologique de France (NS.), 54 (4), 335-366.

Meregalli M, 2017. World Catalogue of the Curculionidae: Lixinae: Cleonini. In: Lyal C.H.C. (Ed.) International Weevil Community Website. Available from: http://weevil.info/content/world-catalogue-curculionidaelixinae-cleonini.

O'Hara JE, Henderson SJ, Wood DM, 2019. Preliminary Checklist of the Tachinidae of the World. Version 1.0. PDF document, 681p. Retrieved from http://www.nadsdiptera.org/Tach/WorldTachs/Checklist/Worldchecklist.html. (Date of access: 20 July 2020).

Richter VA, Gültekin L, Korotyaev BA, 2002. Zeuxia cinerea Meigen New to the Fauna of Northeastern Turkey (Diptera: Tachinidae). Zoosystematica Rossica, 11 (1), 234.

Stireman JO, O'Hara JE, Wood DM, 2006. Tachinidae: Evolution, Behavior and Ecology. Annual Review of Entomology, 51 (1), 525-555.

Ter-Minassian ME, 1988. Weevils of the Subfamily Cleoninae of the Fauna of USSR: Tribe Cleonini. Opredeliteli po Faune SSSR, Izdavaemye Zoologicheskim Institutom Akademii Nauk SSSR, 155, 1-235. 
Tschorsnig HP, Herting B, 1994. Die Raupenfliegen (Diptera: Tachinidae) Mitteleuropas: Bestimmungstabellen und Angaben zur Verbreitung und Ökologie der Einzelnen Arten. Stuttgarter Beiträge zur Naturkunde (A) 506: 1-170. Online Authorized Version of English Translation by Rayner R. \& Raper C.: Tschorsnig H.P. and Herting B., 2001: The Tachinids (Diptera:Tachinidae) of Central Europe: Identification Keys for the Species and Data on Distribution and Ecology, http://tachinidae.org.uk/site/downloads.php. (Date of access: 16 June 2020)..

Tschorsnig HP, 2017. Preliminary Host Catalogue of Palaearctic Tachinidae (Diptera). 480pp www.nadsdiptera.org/Tach/WorldTachs/CatPalHosts/Home.html. (Date of access: 16 June 2020). 